\title{
PIELONEFRITIS AGUDA EN EL EMBARAZO Y SUSCEPTIBILIDAD ANTIMICROBIANA DE UROPATÓGENOS. COMPARACIÓN DE DOS DÉCADAS
}

\author{
Ulises Calderón Ch. ${ }^{1}$, Adriana Doren V. ${ }^{a}$, Magdalena Cruz O. ${ }^{a}$, Jaime Cerda L. ${ }^{2}$, \\ Fernando Abarzúa C. ${ }^{3}$ \\ 1 Programa de Obstetricia y Ginecología, Universidad Nacional de Trujillo, Perú. \\ 2 Departamento de Salud Pública, ${ }^{3}$ Departamento de Obstetricia y Ginecología, Facultad de Medicina, Pontificia \\ Universidad Católica de Chile.
}

a Internas de Medicina, Facultad de Medicina, Pontificia Universidad Católica de Chile.

\section{RESUMEN}

Antecedentes: La infección de tracto urinario (ITU) es la patología infecciosa bacteriana más frecuente del embarazo. Los esfuerzos por lograr una detección precoz y tratamiento adecuado se basan en la toma sistemática de urocultivo al inicio del embarazo, estudiando los agentes causales y la sensibilidad de éstos a los antibióticos recomendados durante la gestación. Objetivo: Describir los cuadros clínicos y microbiológicos de los casos de ITU registrados en embarazadas atendidas en nuestra institución durante el año 2007 y compararlos con los reportes de 1988 y 2001. Métodos: Se revisaron las historias clínicas de 112 embarazadas que cursaron ITU durante 2007, determinando la frecuencia de pielonefritis aguda (PNA), agente infeccioso y susceptibilidad a los antimicrobianos. Se compararon estos datos con los registrados en 2001 y 1988 mediante el cálculo de Odds Ratios. Resultados: La proporción de PNA mostró una tendencia decreciente entre 1988, 2001 y 2007 ( $p<0,001)$. Escherichia coli fue el agente infeccioso más frecuente, sin embargo, su frecuencia disminuyó entre 2001 y 2007 (OR 0,32; IC95\% 0,17-0,58). Se observó un aumento de ITU por Streptococcus agalactiae (SGB) (OR 3,98; 1,85-8,67) durante igual período. La sensibilidad antimicrobiana a ampicilina, gentamicina, nitrofurantoína y cotrimoxazol no presentó diferencias significativas entre 2001-2007. Conclusión: Se documentó un cambio en el perfil clínico y microbiológico de las ITU en embarazadas, reduciéndose la proporción de PNA e ITU por Escherichia coli.

\section{PALABRAS CLAVE: Infección urinaria, embarazo, resistencia antimicrobiana}

\section{SUMMARY}

Background: Urinary tract infection (UTI) is the most frequent bacterial infectious pathology during pregnancy. Efforts to accomplish an early detection and treatment are based on universal urine culture during pregnancy, study of etiologic agents and their susceptibility to antimicrobials prescripted during pregnancy. Objective: To describe the clinical and microbiological characteristics of cases of UTI in pregnant women attended in our institution during 2007 and compare them with the reports of 1988 and 2001. Methods: Clinical records of 112 pregnant women who presented UTI were reviewed, establishing the frequency of acute pyelonephritis (APN), infectious agent and antimicrobial susceptibility. Data were compared statistically with the records of 2001 and 1988 by calculating odds ratios. Results: The proportion of APN showed a decreasing 
trend between 1988, 2001 and $2007(\mathrm{p}<0.001)$. Escherichia coli was the most frequent infectious agent, although its frequency reduced between 2001 and 2007 (OR 0.32; IC95\% 0.17-0.58). On the other hand, the frequency of UTI caused by Streptococcus agalactiae (GBS) increased during the same period of time (OR 3.98; 1.85-8.67). Antimicrobial susceptibility to ampicillin, gentamicin, nitrofurantoin y cotrimoxazole did not present significant differences between 2001-2007. Conclusion: A change in the clinical and microbiological characteristics of UTI in pregnant women was documented. The proportion of APN and UTI caused by Escherichia coli were reduced.

\section{KEY WORDS: Urinary tract infection, pregnancy, antimicrobial resistance}

\section{INTRODUCCIÓN}

La infección del tracto urinario (ITU) es la patología infecciosa bacteriana más frecuente del embarazo (1). Esta puede clasificarse en tres tipos: bacteriuria asintomática (BA) (2-10\%), cistitis aguda (CA) $(1-3 \%)$ y pielonefritis aguda (PNA) $(0,5-1,5 \%)(2)$. La mayoría de los casos de PNA son consecuencia de ITU bajas (BA y CA) no tratadas, recurrentes o tratadas inadecuadamente (2). La relación entre BA en el embarazo con PNA y pronóstico perinatal adverso fue sugerido por primera vez por Kass en 1959, en un estudio randomizado-controlado, el cual demostró que tratando a las gestantes con BA se prevenía el desarrollo de PNA, evitándose más del $20 \%$ de los partos prematuros $(3,4)$.

La PNA es la causa más común de hospitalización no obstétrica durante el embarazo y puede asociarse a dinámica uterina, sepsis materna y síndrome de distrés respiratorio del adulto $(2,5,6)$. La pesquisa sistemática de BA en el embarazo se asocia a una reducción significativa de PNA y de bajo peso al nacer, sin embargo, el impacto sobre la incidencia de prematurez es dudoso (7).

No hay duda que la terapia antimicrobiana es necesaria cuando la ITU se desarrolla durante el embarazo. Las gestantes diagnosticadas con CA o PNA suelen ser tratadas empíricamente antes de conocerse la sensibilidad antimicrobiana de los uropatógenos (8), basándose la elección de la terapia antibiótica en los patrones de sensibilidad establecidos específicamente por cada institución $(2,9)$.

El objetivo de la terapia es mantener la orina estéril durante toda la gestación, sin embargo, la mejor estrategia para lograr este objetivo aún no está establecida. Actualmente no existe consenso acerca del antimicrobiano a elegir, duración de la terapia o del uso profiláctico de antibióticos durante el embarazo (10-13). En países de bajos recursos esta situación es aún más compleja, debido a la carencia de datos referentes a la resistencia a antimicrobianos por bacterias causantes de ITU durante el embarazo, existiendo además poca in- formación en relación al costo, seguridad y eficacia de los nuevos fármacos disponibles. Bajo estas circunstancias es posible que los antibióticos sean prescritos de manera inapropiada, favoreciendo la resistencia a estos medicamentos $(14,15)$.

En 1988 evaluamos la sensibilidad antimicrobiana de uropatógenos encontrados durante el embarazo y la proporción de PNA observada. A partir de esta información, se desarrollaron guías de práctica clínica para el manejo de BA y PNA. En 2001, se hizo una primera reevaluación, la cual mostró una tendencia hacia la reducción de los episodios de PNA y cambios en los patrones de resistencia antimicrobiana (16).

El objetivo de este estudio es describir las tendencias de las características clínicas y microbiológicas de los casos de ITU en embarazadas atendidas en la Institución en 2007, comparándolas con los datos registrados en 1988 y 2001.

\section{PACIENTES Y MÉTODOS}

Se identificaron los casos de ITU en embarazadas ocurridos durante el año 2007 mediante la revisión de las fichas clínicas de las pacientes que controlaron su embarazo en el Policlínico de Obstetricia del Hospital Clínico de la Pontificia Universidad Católica de Chile. Se incluyeron también pacientes atendidas en el servicio de urgencia y hospitalizadas con diagnóstico de PNA. Se definió BA como un recuento mayor a 100.000 unidades formadoras de colonias (UFC) por $\mathrm{mL}$ de orina de segundo chorro, en una paciente asintomática (examen tomado a modo de tamizaje de ingreso a control prenatal). La CA fue descrita como presencia de síntomas urinarios bajos tales como disuria, polaquiuria, tenesmo vesical y urgencia miccional, sin asociación a fiebre, ni dolor lumbar a la puño percusión, más la presencia de un urocultivo positivo con más de $100.000 \mathrm{UFC} / \mathrm{mL}$.

La BA y CA fueron agrupadas bajo el nombre de ITU baja, para efectos de análisis. Por su parte, se definió PNA o ITU alta como un cuadro febril 
asociado a dolor lumbar unilateral, puño percusión positiva, calofríos y orina turbia, con o sin síntomas urinarios bajos y con un urocultivo positivo con más de 100.000 UFC/mL. Para cada embarazada se registró el tipo clínico de ITU (ITU baja vs. alta), agente causal, susceptibilidad antimicrobiana, duración y dosis del antibiótico utilizado, resultado del urocultivo de control y presencia de recaída.

En relación al procesamiento de las muestras de orina, ésta se obtuvo por segundo chorro, sembrándose con asa calibrada en agar sangre y MacConkey con técnica cuantitativa. La susceptibilidad antimicrobiana se analizó a través de concentración inhibitoria mínima (CIM), de acuerdo a la normativa de NCCLS (10). Se estudió sensibilidad de las cepas a ampicilina, cefazolina, cefadroxilo, cefuroxima, gentamicina, cotrimoxazol y nitrofurantoína.

Se compararon los resultados obtenidos en 2007 con aquellos registrados en 2001 y 1988, mediante el cálculo de Odds Ratio (OR) y pueba de Chi-cuadrado para tendencias. Se consideró estadísticamente significativo todo valor $p<0,05$.

\section{RESULTADOS}

Durante 2007 se identificaron 112 embarazadas que presentaron un episodio de ITU, las cuales fueron incluidas en el análisis del cuadro clínico y del tipo de uropatógeno. Para el estudio de susceptibilidad antimicrobiana hubo cuatro casos para los cuales se reportaron cultivos con dos uropatógenos aislados. En estas cuatro pacientes se realizó antibiograma para cada germen, independiente del número de UFC, de modo que para el análisis de los datos de sensibilidad se contó con un universo de 116 informes.

En relación al cuadro clínico, la ITU alta se presentó en $4 / 112$ pacientes (3,6\%), demostrándose una tendencia decreciente de la proporción ITU alta/ITU total al comparar con los años 2001 y 1988 (chi-cuadrado para tendencias $p<0,001$ ) (Tabla I). El uropatógeno más frecuentemente aislado fue $E$. coli $(60 / 116=51,7 \%)$ (Tabla II) demostrándose una reducción significativa en comparación al 77,3\% documentado en 2001 (OR 0,32; IC95\% 0,17-0,58). Le sigue en frecuencia Streptococcus agalactiae $(38 / 116=32,8 \%)$, el cual mostró un aumento significativo en relación al 10,9\% observado en 2001 (OR 3,98; IC 95\% 1,85-8,67). En las cuatro pacientes con PNA aguda en 2007 el microorganismo aislado fue $E$. coli.

La Tabla III resume la frecuencia de resistencia antimicrobiana de todas las cepas en conjunto, correspondiendo el mayor porcentaje a ampicilina
$(21,1 \%)$. En comparación al año 2001 , no se demostraron diferencias significativas en los patrones de resistencia antimicrobiana de ampicilina, gentamicina, nitrofurantoína y cotrimoxazol (únicos antibióticos comparados estadísticamente).

Analizada separadamente, E. coli presentó $30 \%$ de resistencia a ampicilina, $10,5 \%$ a cefadroxilo, $1,6 \%$ a cefazolina y $20,3 \%$ a cotrimoxazol, $\sin$ reportarse resistencia a cefuroximo, gentamicina y nitrofurantoína (Tabla IV). Streptococcus grupo B continúa siendo $100 \%$ sensible a ampicilina.

Finalmente, en relación al seguimiento realizado a las pacientes del año en estudio, 88/112 $(78,6 \%)$ tenían urocultivo de control, resultando positivo en 17/88 (19,3\%). Los microorganismos más frecuentemente aislados fueron E. coli y SGB. En estas pacientes se llevó a cabo un segundo tratamiento, basado en el resultado de sus respectivos antibiogramas.

\section{DISCUSIÓN}

Aunque la incidencia de BA es similar en mujeres embarazadas que en las que no lo están, la prevalencia de PNA es significativamente mayor en gestantes con BA. De hecho, $20-30 \%$ de las BA no tratadas durante el embarazo desarrollarán PNA, complicación grave responsable de distrés respiratorio del adulto y shock séptico en los casos más severos (6). Es por esto que en el primer control prenatal se realiza rutinariamente un urocultivo, cuyo objetivo es reducir la incidencia de esta seria patología. Nuestra serie muestra una reducción significativa de la proporción de PNA en los últimos 20 años, presentándose actualmente sólo en un 3,6\% de los casos reportados, lo que es concordante con la literatura $(7,16)$.

Tabla I

FRECUENCIA DE PIELONEFRITIS AGUDA EN EMBARAZADAS QUE CURSARON ITU. HOSPITAL CLÍNICO UNIVERSIDAD CATÓLICA, 1988-2007

\begin{tabular}{lccr}
\hline & $\begin{array}{r}1988 \\
(\mathrm{n}=110)\end{array}$ & $\begin{array}{c}2001 \\
(\mathrm{n}=110)\end{array}$ & $\begin{array}{r}2007 \\
(\mathrm{n}=112)\end{array}$ \\
\hline Pielonefritis aguda & 29 & 12 & 4 \\
& $(26,3 \%)$ & $(10,9 \%)$ & $(3,6 \%)$ \\
Odds ratio (OR) & 1,00 & 0,34 & 0,10 \\
\hline
\end{tabular}

* Chi-cuadrado para tendencias $p<0,001$ 
Tabla II

PRINCIPALES MICROORGANISMOS

CAUSANTES DE ITU EN EMBARAZADAS. HOSPITAL CLÍNICO UNIVERSIDAD CATÓLICA, 1988-2007

\begin{tabular}{lccc}
\hline Uropatógeno & $\begin{array}{c}1988 \\
(\mathrm{n}=110)\end{array}$ & $\begin{array}{c}2001 \\
(\mathrm{n}=110)\end{array}$ & $\begin{array}{c}2007 \\
(\mathrm{n}=116)\end{array}$ \\
\hline Escherichia coli & 80 & 76,5 & 51,7 \\
Streptococcus agalactiae & 0,0 & 10,8 & 32,7 \\
Staphylococcus coagulasa $(-)$ & 0,0 & 3,6 & 5,2 \\
Enterococcus & 0,0 & 5,4 & 4,4 \\
Klebsiella pneumoniae & 10 & 1,8 & 2,6 \\
Proteus mirabilis & 6 & 0 & 2,6 \\
Enterobacteraerogenes & 4 & 1,8 & 0,8 \\
Total & 100 & 100 & 100 \\
\hline
\end{tabular}

${ }^{*}$ Valores expresados en porcentajes

Tabla III

RESISTENCIA ANTIMICROBIANA DE UROPATÓGENOS EN EMBARAZADAS. HOSPITAL CLÍNICO UNIVERSIDAD CATÓLICA, 1988-2007

\begin{tabular}{|c|c|c|c|c|c|c|}
\hline \multirow{2}{*}{ Antibiótico } & \multicolumn{2}{|c|}{$\begin{array}{l}1988 \\
(n=110)\end{array}$} & \multicolumn{2}{|c|}{$\begin{array}{l}2001 \\
(n=110)\end{array}$} & \multicolumn{2}{|c|}{$\begin{array}{l}2007 \\
(\mathrm{n}=116)\end{array}$} \\
\hline & 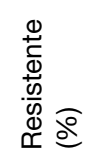 & 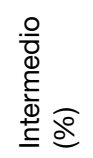 & 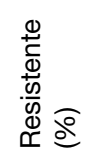 & 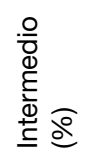 & 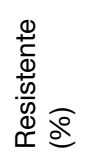 & 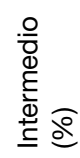 \\
\hline Ampicilina & 35 & 0,0 & 32,6 & 0,9 & 21,1 & 0,0 \\
\hline Cefadroxilo & - & - & - & - & 10 & 0,0 \\
\hline Cefazolina & 0,0 & 0,0 & 8 & 11 & 2,6 & 1,3 \\
\hline Cefuroxima & - & - & - & - & 1,5 & 0,0 \\
\hline Gentamicina & 0,0 & 33 & 3,4 & 0,0 & 1,3 & 2,6 \\
\hline Nitrofurantoína & 8,1 & 0,0 & 9,6 & 0,0 & 3,8 & 3,8 \\
\hline Cotrimoxazol & 20,1 & 0,0 & 31,8 & 0,0 & 18 & 0,0 \\
\hline
\end{tabular}


Tabla IV

\section{RESISTENCIA ANTIMICROBIANA DE ESCHERICHIA COLI. HOSPITAL CLINICO UNIVERSIDAD CATÓLICA, 2007}

\begin{tabular}{lcc}
\hline & \multicolumn{2}{c}{ Escherichia coli } \\
Antibiótico & $\begin{array}{c}\text { Resistente } \\
(\%)\end{array}$ & $\begin{array}{c}\text { Intermedio } \\
(\%)\end{array}$ \\
\hline Ampicilina & 30 & 0,0 \\
Cefadroxilo & 10,5 & 0,0 \\
Cefazolina & 1,6 & 1,6 \\
Cefuroxima & 0,0 & 0,0 \\
Gentamicina & 0,0 & 3,3 \\
Nitrofurantoína & 0,0 & 1,6 \\
Cotrimoxazol & 20,3 & 0,0 \\
\hline
\end{tabular}

La elección del antibiótico para tratar las ITU debe ser guiado por los parámetros locales de resistencia, evidenciados por ensayos clínicos. Una revisión sistemática sobre tratamientos para ITU sintomáticas durante el embarazo concluyó que aunque un tratamiento antibiótico cura efectivamente las ITU durante éste, no hay suficientes datos para recomendar un régimen específico (17).

En el Servicio de Obstetricia de la Universidad Católica aplicamos esta recomendación basándose en los resultados encontrados a nivel local. En 1988 se publicó el primer reporte de los patógenos más frecuentes y su susceptibilidad antimicrobiana (18), el cual nos ha servido como base para los esquemas empíricos aplicados en nuestra institución en la actualidad. Basados en este reporte y en su reevaluación el año 2001 (16), el tratamiento de elección corresponde a cefalosporinas de primera generación, de administración oral en ITU baja (cefadroxilo) y parenteral en el caso de PNA (cefazolina), lo cual ha demostrado bastante éxito al analizar los resultados actuales.

La reducción significativa de la proporción de casos de PNA en los últimos 20 años probablemente se debe a la aplicación sistemática de tamizaje con urocultivo realizado al inicio de los controles prenatales.

En relación a los uropatógenos cultivados, la E. coli mantiene el primer lugar, con un porcentaje similar al reportado en otros estudios a nivel mundial $(6,19-21)$. Blomberg y cols (22), reportaron una frecuencia de sólo 25,2\%, mas en su estudio, hay cepas gramnegativas no identificadas, las cuales sumaban un $21 \%$ del total de casos. Este patógeno continúa siendo el principal agente causante de ITU tanto a nivel mundial como en nuestra institución, probablemente debido a su gran capacidad de invadir células epiteliales mediante adhesinas y hemolisinas. En segundo lugar se encuentra el SGB, agente que ha presentado un aumento estadísticamente significativo con respecto a la proporción de casos reportados el año 2001. Este hecho es relevante desde un punto de vista epidemiológico, pero no así en relación a la terapia, ya que el tratamiento de elección utilizado en la institución tiene buena cobertura para ambos patógenos.

En el análisis de la sensibilidad general de los uropatógenos aislados en nuestra serie, encontramos que no hubo variación significativa en cuanto al patrón de resistencia con respecto al año 2001, siendo similar también a los mostrados en los estudios internacionales (6,19-22). Cabe destacar la buena sensibilidad de la E. coli a las cefalosporinas de primera generación, así como la susceptibilidad del $100 \%$ de los casos de SGB a la ampicilina. Cefuroxima, gentamicina y nitrofurantoína continúan siendo una buena alternativa terapéutica ya que muestran sensibilidad adecuada en el estudio de susceptibilidad. Es probable que este hallazgo se deba a la disminución en su uso como fármacos de primera línea, principalmente en el caso de la nitrofurantoína.

En cuanto al seguimiento realizado a las 112 pacientes del año 2007, se dispuso del urocultivo de control en 88 de ellas. Teniendo en cuenta que cerca del $20 \%$ de los cultivos efectuados en forma posterior al tratamiento resultaron positivos, podemos concluir la importancia de realizar un seguimiento más controlado de las pacientes que cursan con ITU durante el embarazo. Esto refuerza la dificultad para esterilizar la orina en mujeres embarazadas en comparación con las no gestantes a pesar de la sensibilidad in vitro a un antimicrobiano, la dificultad para proponer tratamientos acortados y la obligatoriedad de contar con un urocultivo de control pos tratamiento en esta circunstancia.

\section{CONCLUSIÓN}

Hubo una reducción significativa en la proporción de PNA en las embarazadas durante las últimas dos décadas. Los patógenos involucrados siguen siendo los mismos y la sensibilidad de éstos a los antimicrobianos se mantienen relativamente estable durante este período. 


\section{BIBLIOGRAFIA}

1. Gibbs RS, Sweet RL. Maternal and fetal infectious disorders. In Creasy RK, Resnik R (eds). MaternalFetal Medicine, 4th ed. Philadelphia: W.B. Saunders Company, 1999:659-724.

2. Duff $P$. Urinary tract infections. Prim Care Update $\mathrm{Ob}$ Gyn 1994;1:12-16.

3. Kass EH. The role of asymptomatic bacteriuria in the pathogenesis of pyelonephritis. In: Quinn EL, Kass EH (eds). Biology of pyelonephritis. Boston: Little, Brown and Co., 1960:399-412.

4. Smaill FM. Asymptomatic bacteriuria in pregnancy. Best Pract Res Clin Obstet Gynaecol 2007;21(3):43950.

5. Duff P. Pyelonephritis in pregnancy. Clin Obstet Gynecol 1984;27:17-31.

6. Jamie WE, Edwards RK, Duff P. Antimicrobial susceptibility of Gram-negative uropathogens isolated from obstetric patients. Infect Dis Obstet Gynecol 2002;10(3):123-6.

7. Smaill FM, Vazquez JC. Antibiotics for asymptomatic bacteriuria in pregnancy. Cochrane Database of Systematic Reviews 2007, Issue 2. Art. No.: CD000490.

8. Vercaigne LM, Zhanel GG. Recommended treatment for urinary tract infection in pregnancy. Ann Pharmacother 1994;28:248-51.

9. Dyer IE, Sankary TM, Dawson JA. Antibiotic resistance in bacterial urinary tract infections, 1991 to 1997. West J Med 1998;169(5):265-8.

10. National Committee for Clinical Laboratory Standards. Performance standards for antimicrobial susceptibility testing. XII Informational Supplement. M100-S12. Wayne, Pennsylvania, NCCLS, 2002.

11. Macejko A, Schaeffer A. Asymptomatic bacteriuria and symptomatic urinary tract infections during pregnancy. Urol Clin N Am.2007;34(1):35-42.

12. Duff P. Antibiotic selection in obstetrics: making costeffective choices. Clin Obstet Gynecol 2002;45:5972.
13. Mandal P, Kapil A, Goswami K, Das B, Dwivedi SN. Uropathogenic Escherichia coli causing urinary tract infections. Indian J Med Res 2001;114:207-11.

14. Fluit AC, Schmitz FJ. Bacterial resistance in urinary tract infection: how to stem the tide. Expert Opin Pharmacother 2001; 2(5): 813-8.

15. Mathai E, Thomas RJ, Chandy S, Mathai M, Bergstrom S. Antimicrobials for the treatment of urinary tract infection in pregnancy: practices in southern India. Pharmacoepidemiol Drug Saf 2004;13:645-52.

16. Abarzúa $F$, Zajer $C$, Donoso $B$, et al. Reevaluación de la sensibilidad antimicrobiana de patógenos urinarios en el embarazo. Rev Chil Obstet Ginecol 2002;67:22631.

17. Vazquez JC, Villar J. Treatments for symptomatic urinary tract infections during pregnancy. Cochrane Database of Systematic Reviews 2003, Issue 4. Art. No.: CD002256.

18. González P, Correa R, Montiel F. Tratamiento de la infección urinaria durante el embarazo: experiencia en 110 pacientes. Rev Med Chile 1988;116:895-900.

19. Quiroga G, Robles R, Ruelas A, Gómez A. Bacteriuria asintomática en mujeres embarazadas. Una amenaza subestimada. Rev Med Inst Mex Seguro Soc 2007;45: 169-72.

20. Hill J, Sheffield J, Mclntire D, Wendel G. Pyelonephritis in pregnancy. Obstet Gynecol 2005;105:18-23.

21. Hernández F, López J, Rodríguez J, Peralta M, Rodríguez R, Ortíz A. Frecuencia de bacteriuria asintomática en embarazadas y sensibilidad antimicrobiana in vitro de los uropatógenos. Ginecol Obstet Mex 2007;75:325-31.

22. Blomberg B, Evjen Olsen B, Gudmund Hinderaker S, et al. Antimicrobial resistance in urinary bacterial isolates from pregnant women in rural Tanzania: Implications for public health. Scand J Infect Dis 2005;37:2628. 